\title{
Utilización de las bases de datos de patentes como instrumento de vigilancia tecnológica
}

\author{
Por Antonio Hidalgo-Nuchera, Santiago Iglesias-Pradas y Ángel Hernández-García
}

\begin{abstract}
Resumen: Ante la importancia que adquieren la innovación y el desarrollo tecnológico en la economía de un país, es fundamental contar con indicadores que permitan medir el grado de innovación de sus empresas. Los indicadores relativos a las patentes proporcionan una excelente medida de esta capacidad y son una destacada fuente de información para organizaciones innovadoras, aunque se han llevado a cabo pocos estudios que midan esta utilidad. Se plantea, en base a un análisis realizado a lo largo de 2007, el nivel de uso de las bases de datos de patentes orientado a la vigilancia tecnológica por parte de más de medio millar de empresas españolas que presentaron al menos una patente en el período 1988-1998. Los resultados se han analizado tanto globalmente como en función del tamaño y sector de actividad económica de la empresa.
\end{abstract}

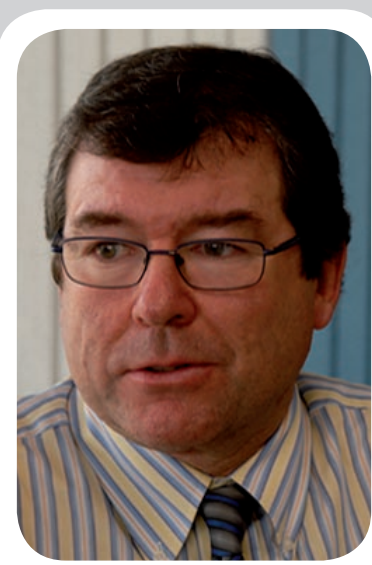

Antonio Hidalgo-Nuchera es profesor titular de Organización de empresas en la Escuela Técnica Superior de Ingenieros Industriales de la Univ. Politécnica de Madrid y director del grupo de investigación en Innovación, Propiedad Industrial y Política Tecnológica (Innopro). Ha participado en diferentes proyectos financiados por la Comisión Europea y organismos nacionales sobre innovación tecnológica, transferencia de tecnología y desarrollo de políticas tecnológicas.

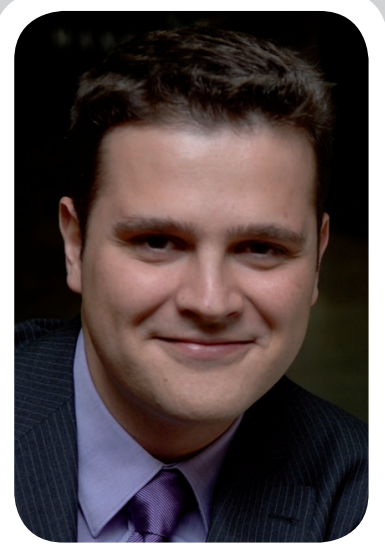

Santiago Iglesias-Pradas es profesor de Organización de empresas de la Universidad Politécnica de Madrid. Imparte asignaturas de grado y postgrado en las áreas de dirección de empresas y de tecnologías y sistemas de información empresariales en distintas universidades. Es doctor ingeniero de telecomunicación y MBA por la UPM, ha participado en diferentes proyectos de investigación nacionales e internacionales y pertenece al grupo Innovación, Propiedad Industrial y Política Tecnológica (Innopro).



Ángel Hernández-García es ingeniero de telecomunicación por la Univ. Politécnica de Madrid y trabaja como investigador doctoral en la misma universidad. Sus líneas de investigación están orientadas a la innovación tecnológica, la aplicación de modelos de aceptación de la tecnología en mercados electrónicos, caracterización de contenidos digitales en dispositivos móviles y análisis de factores de influencia en el rendimiento académico en sistemas e-learning.

Palabras clave: Patentes, Innovación, Vigilancia tecnológica, Bases de datos, Servicios de información, Indicadores.

Title: Use of patent databases as a tool for technology watch

Abstract: Given the importance of innovation and technology development in a country's economy, there has been an increasing need for selecting indicators to measure the degree of innovation of its companies. The indicators related to patents offer an excellent measure of this capability and can be used as an important source of information by innovative organizations, although the studies assessing this utility are scarce. This study, conducted throughout 2007, proposes a technology watch oriented analysis of the level of use of patent databases from more than 500 Spanish companies that have issued at least one patent in the period 1988-1998, followed by discussion of the study results. The analysis took into account global data, company size and sector of activity.

Keywords: Patents, Innovation, Technology watch, Databases, Information services, Indicators.

Hidalgo-Nuchera, Antonio; Iglesias-Pradas, Santiago; Hernández-García, Ángel. "Utilización de las bases de datos de patentes como instrumento de vigilancia tecnológica". El profesional de la información, 2009, septiembre-octubre, v. 18 , n. 5, pp. 511-519.

DOI: 10.3145/epi.2009.sep.04

\section{Introducción}

Un factor destacado en la competitividad de la economía de un país es su capacidad de estimular la innovación y el desarrollo tecnológico. La innovación constituye una de las claves del crecimiento económico a largo plazo, por lo que es necesario identificar en qué medida es de origen nacional o transferida mediante licencias, importaciones, imitaciones o inversiones extranjeras directas. 
Este interés se ha consolidado debido a que los elementos que intervienen en el proceso de desarrollo tecnológico, cuando éste se estudia desde la óptica de la acumulación de conocimientos y el aprendizaje, abarcan un campo de variables mucho más amplio (Freeman; Soete, 1982; Dosi, 1988). También ha contribuido a ello la consideración, a partir de mediados de los ochenta, de la innovación como un proceso independiente e interactivo (Kline; Rosenberg, 1986), con lógica propia y al que contribuyen de forma relevante pero no exclusiva, las tareas propias de investigación. El Manual de Oslo (OCDE, 2005) ha mejorado la interpretación de la medición de iniciativas de innovación tecnológica, y ha propiciado la elaboración de las recientes Encuestas de Innovación Tecnológica del CIS apoyadas por la Comisión Europea.

Las patentes constituyen los indicadores del output de la organización que aportan la información más relevante sobre el conjunto del proceso de innovación tecnológica. Desde los primeros trabajos realizados utilizando patentes como indicadores de actividades tecnológicas (Schmookler, 1966), el uso de la información que contienen ha experimentado un cambio más que significativo, de forma que en la actualidad es casi imposible encontrar trabajos sobre los procesos de innovación o las capacidades tecnológicas de cualquier organización (empresa, universidad, centro de investigación) sin que se utilicen las patentes como indicadores.

No obstante, este uso lleva asociado de forma implícita un conjunto de dificultades que es necesario tener en cuenta (Basberg, 1987; Pavitt, 1998; Archibugi, 1992):

- Sólo una parte de las innovaciones se patentan, ya que hay otros mecanismos diferentes para proteger las invenciones, por ejemplo, el secreto industrial.

- Algunas invenciones no son patentables, o porque la legislación las excluye expresamente o porque no cumplen alguno de los requisitos necesarios (novedad, actividad inventiva, aplicación industrial).

- En determinados sectores el ciclo de vida de los productos es muy corto y no es interesante patentar.

En todo caso y a pesar de las dificultades enumeradas, son muchas las ventajas que conlleva utilizar las patentes como indicadores de la actividad tecnológica de la organización, ya que representan la culminación de un esfuerzo por parte de ésta que pone de manifiesto no sólo la intensidad de las labores inventivas, sino la capacidad de implementar las propias competencias tecnológicas. Entre estas ventajas se encuentra el hecho de que se dispone de series de datos que cubren períodos de tiempo relativamente largos y que contie- nen información detallada (incluyendo los datos de la empresa y del sector industrial). Estos datos pueden ser utilizados para analizar la actividad nacional de innovación con un elevado nivel de precisión.

\section{"Las patentes aportan la información más relevante sobre el conjunto del proceso de innovación tecnológica"}

Esta información tecnológica se encuentra en las bases de datos de organismos nacionales e internacionales como la Oficina Española de Patentes y Marcas (Oepm), la Oficina Europea de Patentes (EPO), la Oficina Norteamericana de Patentes (Uspto), la Organización Mundial de la Propiedad Intelectual (Ompi), etc. El análisis de los datos bibliográficos extraídos de un documento de patente puede proporcionar información muy valiosa. Por ejemplo, un análisis de la relación entre la tasa de denegación de patentes frente al número de solicitudes realizadas y sobre las causas que provocaron dicha denegación (qué requisito de patentabilidad falló), puede aportar una excelente información acerca de hasta qué punto las empresas, las universidades y los centros de investigación llevan a cabo labores de vigilancia tecnológica y cómo utilizan esta información antes de aportar fondos a proyectos de investigación. Asimismo, este análisis hace posible la obtención de información relacionada con otro conjunto de aspectos, entre los que destacan:

- La detección de las empresas que, debido a sus altas tasas de concesión frente a solicitudes, son compañías ejemplares en su sector de actividad en la utilización de la propiedad industrial como forma de proteger la innovación, así como en el uso de fuentes de información tecnológica para evitar la duplicidad de esfuerzos.

- Cómo y cuándo las compañías utilizan las bases de datos de patentes, es decir, se puede medir hasta qué punto la información de las patentes es utilizada antes y durante la investigación.

- La calidad de una patente en función de los requisitos de patentabilidad.

\section{Objetivos y metodología}

Teniendo en cuenta estas consideraciones, la finalidad del proyecto fue analizar el grado en que el colectivo de empresas españolas con al menos una patente concedida y que fuera solicitada en el período 1988- 
1998 ha utilizado las bases de datos de patentes hasta el año 2007 como instrumento de vigilancia tecnológica. Para ello, se ha tomado como punto de partida la siguiente información:

- Patentes con prioridad española (o sea, España primer país donde se solicita la patente) que, habiendo sido solicitadas en la Oficina Europea de Patentes entre los años 1988 y 1998, han sido concedidas hasta la fecha de realización del estudio (se ha de tener en cuenta que la concesión de estas patentes conlleva un plazo medio de aproximadamente 5 años). En este colectivo se incluye también a empresas e inventores extranjeros que protegen sus invenciones en España en primer lugar, bien por estrategia de protección o bien porque la invención es realizada en España.

- Solicitudes de patentes realizadas por sociedades españolas en el período 1988-1998 que han sido concedidas hasta 2007 (sin prioridad española).

\section{"Son muchas las ventajas que conlleva utilizar las patentes como indicadores de la actividad tecnológica"}

Para la realización del análisis se han utilizado los datos procedentes de la Oficina Europea de Patentes, ya que sus bases de datos Epat y Espacenet contienen información con la precisión y calidad suficiente para los propósitos fijados. La primera ofrece información básica de las solicitudes (fechas, solicitantes, organismos, etc.) y facilita la consulta de más de 830.000 referencias. Por su parte, Espacenet contiene los datos bibliográficos y los documentos completos en formato pdf de las patentes publicadas en los últimos años en cualquier estado miembro de la Organización Europea de Patentes, así como los de la Oficina Europea de Patentes y de la Organización Mundial de la Propiedad Intelectual. Su volumen es de unos 30 millones de documentos.

Del análisis de las bases de datos Epat y Espacenet, y de acuerdo con los criterios de selección mencionados, se ha obtenido para el período 1988-1998 un total de 5.046 patentes concedidas correspondientes a 546 empresas. La distribución de estas patentes en función del tipo de información referenciada ha sido la siguiente:

- concedidas con prioridad española o solicitante español: 4.197 registros;

- concedidas a inventores españoles fuera de España: 849 registros.
Para llevar a cabo el análisis se diseñó un cuestionario en el que se solicitaba a las empresas que realizaran una valoración cualitativa sobre el uso que hacen de los servicios de información sobre patentes mediante un conjunto de nueve preguntas dirigidas a los responsables del área tecnológica de la organización:

a. ¿Cuál es la importancia de las patentes obtenidas por su empresa para su negocio?

b. ¿Utiliza o dispone su empresa de servicios de información sobre patentes?

c. ¿Con qué frecuencia utiliza su empresa estos servicios?

d. ¿Para qué se utilizan estos servicios de información?

e. ¿Cuáles son los servicios de información normalmente utilizados?

f. En caso de acceder directamente a información sobre patentes, ¿puede especificar qué proveedores de bases de datos o servicios en internet utiliza?

g. ¿Piensa su empresa comenzar a utilizar o incrementar la utilización de los documentos de patentes como fuente de información?

h. ¿Podrían existir factores que impulsasen a su empresa a hacer más efectiva la búsqueda de información en las patentes?

i. ¿Son importantes para el avance de su empresa otras formas de protección de la propiedad industrial?

El cuestionario fue enviado a las 546 empresas españolas, obteniéndose 123 respuestas, lo que representa el 22,5\% de los cuestionarios enviados. El porcentaje más alto $(58,5 \%)$ corresponde a compañías de más de 100 empleados, lo que resulta lógico desde la perspectiva de la gestión innovadora y de las capacidades tecnológicas, pues son las que poseen mayor nivel de recursos (tabla 1$)$.

\begin{tabular}{|l|c|c|c|c|c|}
\hline No empleados & $<\mathbf{1 0}$ & $\begin{array}{c}\mathbf{1 0}- \\
\mathbf{5 0}\end{array}$ & $\begin{array}{c}\mathbf{5 1 -} \\
\mathbf{1 0 0}\end{array}$ & $\begin{array}{c}\mathbf{1 0 1 -} \\
\mathbf{2 5 0}\end{array}$ & $\mathbf{>} \mathbf{2 5 0}$ \\
\hline$\%$ respuestas & 9,7 & 13,0 & 18,8 & 21,1 & 37,4 \\
\hline
\end{tabular}

Tabla 1. Distribución de las respuestas según el número de empleados (\%)

En función de los sectores económicos, los datos obtenidos reflejan que el mayor nivel de respuesta se concentra en química-farmacia e ingeniería mecánica y maquinaria (tabla 2). Este resultado es consistente con el hecho de que estas industrias están condicionadas por la frecuente aparición de rupturas tecnológicas que implican la necesidad de patentar para protegerse de las mismas (Hidalgo; Molero, 2009). 


\begin{tabular}{|c|c|c|c|c|c|c|c|}
\hline \multirow{2}{*}{ Área } & \multirow{2}{*}{$\begin{array}{l}\text { Número de } \\
\text { empresas }\end{array}$} & \multirow{2}{*}{$\begin{array}{c}\text { Porcentaje } \\
(\%)\end{array}$} & \multicolumn{5}{|c|}{ Tamaño (\%) } \\
\hline & & & $<10$ & $10-50$ & $51-100$ & $101-250$ & $>250$ \\
\hline Ingeniería civil, bienes de consumo & 21 & 17 & 14 & 0 & 29 & 29 & 29 \\
\hline Electricidad-electrónica & 19 & 15 & 11 & 11 & 26 & 16 & 37 \\
\hline Ingeniería de proceso & 7 & 6 & 0 & 29 & 14 & 0 & 57 \\
\hline Ingeniería mecánica y maquinaria & 34 & 28 & 15 & 18 & 9 & 18 & 41 \\
\hline Instrumentación & 6 & 5 & 33 & 0 & 0 & 33 & 33 \\
\hline Química-farmacia & 36 & 29 & 0 & 17 & 22 & 25 & 36 \\
\hline Total & 123 & 100 & 10 & 13 & 19 & 21 & 37 \\
\hline
\end{tabular}

Tabla 2. Distribución de las respuestas según el sector económico

(para mayor claridad los porcentajes que figuran en las tablas de este artículo se han redondeado)

\section{Resultados obtenidos}

\subsection{Importancia de las patentes obtenidas para el negocio}

De entre las seis opciones que se plantearon a las empresas para que indicaran su importancia a la hora de tomar una decisión para solicitar una patente, éstas consideraron como más crítica la relativa a que la patente es un instrumento de defensa de la propiedad industrial, en el 70\% de las respuestas. Consideran con una importancia media la opción que indica que la patente proporciona mayor capacidad de negociación con otras compañías (42\%), mientras que el resto de posibilidades son consideradas de forma mayoritaria como poco relevantes. Es de destacar que las empresas no consideran la patente como un mecanismo válido para fomentar la exportación de tecnología ni para llevar a cabo su licencia y obtener más ingresos. La tabla 3 refleja el resumen de estas opciones con la valoración mejor considerada (alta-3; media-2; baja-1).

Atendiendo al tamaño de las sociedades, la tabla 4 refleja para cada tipo la opción más valorada, destacando la importancia que conceden las grandes ( $>250$ empleados) a la patente como elemento que ayuda a la exportación de tecnología y a la formalización del conocimiento, factor este último que es muy bien valorado por las que tienen entre 11 y 50 empleados. Por su parte, las microempresas (5-10 empleados) valoran de forma positiva la patente como factor crítico de exportación.

El análisis de esta variable en función del sector económico al que pertenece la organización (tabla 5) pone de relieve que los sectores electricidad-electrónica, ingeniería de proceso, ingeniería mecánica y maquinaria, e ingeniería civil-bienes de consumo presentan

\begin{tabular}{|l|c|c|}
\hline \multicolumn{1}{|c|}{ Opciones } & Importancia & \% \\
\hline $\begin{array}{l}\text { Instrumento de defensa de la } \\
\text { propiedad industrial }\end{array}$ & Alta & 70 \\
\hline $\begin{array}{l}\text { Proporcionar a la empresa mayor } \\
\text { capacidad para solicitar créditos }\end{array}$ & Baja & 77 \\
\hline $\begin{array}{l}\text { Proporcionar a la empresa mayor } \\
\text { capacidad de negociación con } \\
\text { otras empresas }\end{array}$ & Media & 42 \\
\hline $\begin{array}{l}\text { Permitir a la empresa exportar } \\
\text { tecnología }\end{array}$ & Baja & 37 \\
\hline $\begin{array}{l}\text { Licenciar la patente para obtener } \\
\text { mayores ingresos }\end{array}$ & Baja & 53 \\
\hline $\begin{array}{l}\text { Formalizar el conocimiento de la } \\
\text { empresa }\end{array}$ & Baja & 38 \\
\hline
\end{tabular}

Tabla 3. Importancia de las patentes obtenidas por la empresa para su negocio

\begin{tabular}{|c|c|c|c|c|c|}
\hline Opciones & 5-10 & 11-50 & 51-100 & $\begin{array}{l}101- \\
250\end{array}$ & $>250$ \\
\hline Instrumento de defensa de la propiedad industrial & $\begin{array}{c}78 \\
(\text { alta })\end{array}$ & $\begin{array}{c}56 \\
(\text { alta })\end{array}$ & $\begin{array}{c}57 \\
(\text { alta })\end{array}$ & $\begin{array}{c}84 \\
(\text { alta })\end{array}$ & $\begin{array}{c}71 \\
\text { (alta) }\end{array}$ \\
\hline Proporcionar a la empresa mayor capacidad para solicitar créditos & $\begin{array}{c}78 \\
\text { (baja) }\end{array}$ & $\begin{array}{c}69 \\
\text { (baja) }\end{array}$ & $\begin{array}{c}70 \\
\text { (baja) }\end{array}$ & $\begin{array}{c}80 \\
\text { (baja) }\end{array}$ & $\begin{array}{c}80 \\
\text { (baja) }\end{array}$ \\
\hline $\begin{array}{l}\text { Proporcionar a la empresa mayor capacidad de negociación con } \\
\text { otras empresas }\end{array}$ & $\begin{array}{c}44 \\
\text { (media) }\end{array}$ & $\begin{array}{c}44 \\
\text { (baja) }\end{array}$ & $\begin{array}{c}39 \\
\text { (media) }\end{array}$ & $\begin{array}{c}36 \\
\text { (media) }\end{array}$ & $\begin{array}{c}50 \\
\text { (media) }\end{array}$ \\
\hline Permitir a la empresa exportar tecnología & $\begin{array}{c}44 \\
\text { (alta) }\end{array}$ & $\begin{array}{c}44 \\
(\text { baja })\end{array}$ & $\begin{array}{c}57 \\
\text { (baja) } \\
\end{array}$ & $\begin{array}{c}40 \\
\text { (baja) }\end{array}$ & $\begin{array}{c}36 \\
\text { (media) }\end{array}$ \\
\hline Licenciar la patente para obtener mayores ingresos & $\begin{array}{c}33 \\
\text { (baja) }\end{array}$ & $\begin{array}{c}44 \\
\text { (baja) }\end{array}$ & $\begin{array}{c}57 \\
\text { (baja) }\end{array}$ & $\begin{array}{c}60 \\
\text { (baja) }\end{array}$ & $\begin{array}{c}55 \\
\text { (baja) }\end{array}$ \\
\hline Formalizar el conocimiento de la empresa & $\begin{array}{c}67 \\
\text { (baja) }\end{array}$ & $\begin{array}{c}38 \\
\text { (alta) }\end{array}$ & $\begin{array}{c}39 \\
\text { (baja) }\end{array}$ & $\begin{array}{c}40 \\
\text { (media) }\end{array}$ & $\begin{array}{c}32 \\
\text { (media) }\end{array}$ \\
\hline
\end{tabular}

Tabla 4. Importancia de las patentes en función del tamaño de la empresa (\%) 


\begin{tabular}{|l|c|c|c|c|c|c|}
\hline \multicolumn{1}{|c|}{ Opciones } & $\begin{array}{c}\text { Bienes de } \\
\text { consumo, } \\
\text { ingeniería } \\
\text { civil }\end{array}$ & $\begin{array}{c}\text { Electricidad- } \\
\text { electrónica }\end{array}$ & $\begin{array}{c}\text { Ingeniería } \\
\text { de } \\
\text { proceso }\end{array}$ & $\begin{array}{c}\text { Ingeniería } \\
\text { mecánica y } \\
\text { maquinaria }\end{array}$ & $\begin{array}{c}\text { Instrumen- } \\
\text { tación }\end{array}$ & $\begin{array}{c}\text { Química- } \\
\text { farmacia }\end{array}$ \\
\hline $\begin{array}{l}\text { Instrumento de defensa de la } \\
\text { propiedad industrial }\end{array}$ & $\begin{array}{c}71 \\
\text { (alta) }\end{array}$ & $\begin{array}{c}74 \\
\text { (alta) }\end{array}$ & $\begin{array}{c}71 \\
\text { (alta) }\end{array}$ & $\begin{array}{c}62 \\
\text { (alta) }\end{array}$ & $\begin{array}{c}67 \\
\text { (alta) }\end{array}$ & $\begin{array}{c}75 \\
\text { (alta) }\end{array}$ \\
\hline $\begin{array}{l}\text { Proporcionar a la empresa mayor } \\
\text { capacidad para solicitar créditos }\end{array}$ & $\begin{array}{c}86 \\
\text { (baja) }\end{array}$ & $\begin{array}{c}84 \\
\text { (baja) }\end{array}$ & $\begin{array}{c}57 \\
\text { (baja) }\end{array}$ & $\begin{array}{c}74 \\
\text { (baja) }\end{array}$ & $\begin{array}{c}50 \\
\text { (media) }\end{array}$ & $\begin{array}{c}86 \\
\text { (baja) }\end{array}$ \\
\hline $\begin{array}{l}\text { Proporcionar a la empresa mayor } \\
\text { capacidad de negociación con otras } \\
\text { empresas }\end{array}$ & $\begin{array}{c}52 \\
\text { (media) }\end{array}$ & $\begin{array}{c}42 \\
\text { (media) }\end{array}$ & $\begin{array}{c}29 \\
\text { (media) }\end{array}$ & $\begin{array}{c}47 \\
\text { (media) }\end{array}$ & $\begin{array}{c}50 \\
\text { (alta) }\end{array}$ & $\begin{array}{c}36 \\
\text { (media) }\end{array}$ \\
\hline $\begin{array}{l}\text { Permitir a la empresa exportar } \\
\text { tecnología }\end{array}$ & $\begin{array}{c}38 \\
\text { (baja) }\end{array}$ & $\begin{array}{c}53 \\
\text { (baja) }\end{array}$ & $\begin{array}{c}43 \\
\text { (baja) }\end{array}$ & $\begin{array}{c}41 \\
\text { (baja) }\end{array}$ & $\begin{array}{c}83 \\
\text { (alta) }\end{array}$ & $\begin{array}{c}44 \\
\text { (media) }\end{array}$ \\
\hline $\begin{array}{l}\text { Licenciar la patente para obtener } \\
\text { mayores ingresos }\end{array}$ & $\begin{array}{c}71 \\
\text { (baja) }\end{array}$ & $\begin{array}{c}58 \\
\text { (baja) }\end{array}$ & $\begin{array}{c}43 \\
\text { (baja) }\end{array}$ & $\begin{array}{c}59 \\
\text { (baja) }\end{array}$ & $\begin{array}{c}50 \\
\text { (alta) }\end{array}$ & $\begin{array}{c}39 \\
\text { (baja) }\end{array}$ \\
\hline $\begin{array}{l}\text { Formalizar el conocimiento de la } \\
\text { empresa }\end{array}$ & $\begin{array}{c}57 \\
\text { (baja) }\end{array}$ & $\begin{array}{c}47 \\
\text { (baja) }\end{array}$ & $\begin{array}{c}29 \\
\text { (baja) }\end{array}$ & $\begin{array}{c}38 \\
\text { (media) }\end{array}$ & $\begin{array}{c}33 \\
\text { (alta) }\end{array}$ & $\begin{array}{c}33 \\
\text { (alta) }\end{array}$ \\
\hline
\end{tabular}

Tabla 5. Importancia de las patentes en función del sector económico (\%)

una estructura de comportamiento semejante, caracterizada por la mayor relevancia del uso de la patente como instrumento de defensa de la propiedad industrial y la consideración de que ésta proporciona mayor capacidad de negociación con otras empresas. En los sectores de instrumentación y química-farmacia las compañías conceden además una gran importancia a la patente como elemento que permite la formalización del conocimiento de la empresa y la exportación de tecnología, lo que se debe en gran medida al mayor tamaño relativo de las sociedades que configuran estos sectores.

\subsection{Uso de servicios de información sobre patentes por la empresa}

Las respuestas obtenidas sobre uso o disponibilidad de algún servicio de información sobre patentes por parte de las empresas son afirmativas en su mayoría (90\%), lo que refleja el alto nivel de interés en acceder a la información contenida en los documentos de patentes.

\section{"Se concede una gran importancia a las patentes, fundamentalmente como instrumento de defensa de la propiedad industrial"}

Respecto al tamaño de la empresa, las que tienen más de 51 empleados son las que más utilizan estos servicios, con un elevado porcentaje de respuestas afirmativas (por encima del 93\%). Las organizaciones con menos de 50 empleados tienen un nivel de respuesta positiva del $75 \%$, lo que resulta importante para este colectivo y pone de relieve el aumento del uso de la patente como instrumento de vigilancia tecnológica.

\subsection{Frecuencia de utilización de los servicios de in- formación sobre patentes}

El conocimiento de la frecuencia en el uso de servicios de información sobre patentes por parte de las sociedades constituye un indicador del interés que tienen en actualizar su nivel tecnológico y acceder a información crítica para la evolución de su actividad, así como de la aceptación de una cultura basada en la vigilancia tecnológica (Escorsa; Maspons, 2001).

El análisis de las respuestas obtenidas pone de manifiesto que el 50\% de las empresas utilizan los servicios de información sobre patentes con una frecuencia mensual (lo que puede considerarse suficiente), mientras que tan sólo el 6\% hacen un uso poco frecuente de estos servicios (frecuencia inferior a un año). El resto categorizan la frecuencia de sus búsquedas en semanal (16\%) y anual (21\%). Por su parte, el análisis por sectores económicos muestra que en todos ellos la media relativa a la frecuencia de búsqueda más usada es de carácter mensual.

En función del tamaño de la empresa se observa que las de más de 51 empleados llevan a cabo sus procesos de búsqueda con una frecuencia mensual, mientras que esa frecuencia es menor para las más pequeñas, lo cual es coherente con el menor nivel de recursos de este colectivo (tabla 6).

\begin{tabular}{|l|c|c|c|c|}
\hline \multirow{2}{*}{$\begin{array}{l}\text { Tamaño de } \\
\text { la empresa }\end{array}$} & \multicolumn{4}{|c|}{ Frecuencia de uso (\%) } \\
\cline { 2 - 5 } & Semanal & Mensual & Anual & >Anual \\
\hline $5-10$ & 11 & 33 & 11 & 22 \\
\hline $11-50$ & 0 & 25 & 44 & 6 \\
\hline $51-100$ & 9 & 65 & 26 & 0 \\
\hline $101-250$ & 24 & 52 & 16 & 0 \\
\hline$>250$ & 21 & 59 & 14 & 2 \\
\hline
\end{tabular}

Tabla 6. Frecuencia de uso de los servicios de información sobre patentes en función del tamaño de la empresa 


\subsection{Finalidad de los servicios de información sobre patentes}

Del conjunto de compañías que afirman utilizar servicios de información sobre patentes, el 65\% lo hace para realizar búsquedas previas al comienzo de un proyecto de I+D en su propia empresa evitando invertir en algo ya existente. Un porcentaje similar responde que los utiliza también para informarse sobre los avances de los competidores $(63 \%)$ y para comprobar si una invención que la empresa ha realizado, está ya hecha con anterioridad (59\%). Estas actuaciones se complementan además con el uso de las patentes para informarse acerca de nuevos avances tecnológicos o ideas innovadoras (tabla 7). En el lado opuesto se encuentran las respuestas que hacen referencia a que los servicios de información sobre patentes apenas son utilizados para llevar a cabo la divulgación de las innovaciones y solventar un problema tecnológico concreto de la empresa.

Del análisis de las respuestas tanto en función del número de empleados de las empresas como de los sectores económicos a los que pertenecen se deduce que, en general, el uso de los servicios de información sobre patentes se realiza fundamentalmente para conseguir los tres fines mejor valorados: informarse acerca de los avances de los competidores, realizar búsquedas previas al comienzo de un proyecto de I+D y comprobar la existencia de una invención realizada.

\begin{tabular}{|l|c|}
\hline \multicolumn{1}{|c|}{ Finalidad } & \% \\
\hline $\begin{array}{l}\text { Comprobar si existe una invención que ha } \\
\text { realizado la empresa }\end{array}$ & 60 \\
\hline $\begin{array}{l}\text { Informarse acerca de nuevos avances } \\
\text { tecnológicos o ideas innovadoras }\end{array}$ & 51 \\
\hline $\begin{array}{l}\text { Informarse acerca de los avances de los } \\
\text { competidores }\end{array}$ & 63 \\
\hline $\begin{array}{l}\text { Solventar un problema tecnológico concreto } \\
\text { de la empresa }\end{array}$ & 14 \\
\hline $\begin{array}{l}\text { Obtener información del mercado } \\
\text { Realizar búsquedas previas al comienzo de } \\
\text { un proyecto de I+D en la empresa evitando } \\
\text { invertir en algo ya existente }\end{array}$ & 65 \\
\hline Divulgar las innovaciones realizadas & 9 \\
\hline
\end{tabular}

Tabla 7. Utilización de los servicios de información sobre patentes

\subsection{Servicios de información sobre patentes más utilizados}

En la actualidad hay diversas fuentes de acceso a la información de patentes, por lo que las empresas pueden diseñar su propia estrategia de búsqueda en base a sus necesidades y diversificar su acción. Las respuestas ponen de manifiesto que las empresas suelen utilizar un número medio de 1,8 fuentes.
De las diferentes opciones posibles, la mayor parte de las organizaciones encuestadas utilizan un agente de la propiedad industrial como principal fuente de información (60\%), lo que probablemente refleja también el uso de los servicios de estos agentes para preparar las solicitudes de patentes. Esta tendencia se observa para todas las empresas, a excepción de las más pequeñas (5-10 empleados) que prefieren utilizar los servicios que proporciona la Oficina Española de Patentes y Marcas (56\%), lo que se explica por los mayores costes que implica utilizar los servicios profesionales de los agentes de la propiedad industrial.

Las siguientes opciones más utilizadas para acceder a la información contenida en las patentes son la Oficina Española de Patentes y Marcas (40\%) y el acceso directo por la propia empresa desde internet, cdrom o bases de datos de patentes online. El resto de posibilidades no tiene un uso extendido, tal y como se refleja en la tabla 8 , destacando en este aspecto el relativamente escaso papel que desempeñan las agencias regionales de desarrollo, los centros tecnológicos y las asociaciones empresariales en este proceso.

\begin{tabular}{|l|c|}
\hline \multicolumn{1}{|c|}{ Servicios de información } & $\%$ \\
\hline Oficina Española de Patentes y Marcas & 40 \\
\hline Agente de la propiedad industrial & 60 \\
\hline $\begin{array}{l}\text { Directamente por la empresa (internet, } \\
\text { cd-rom, etc.) }\end{array}$ & 36 \\
\hline $\begin{array}{l}\text { Directamente por la empresa o desde una } \\
\text { biblioteca de documentos de patentes }\end{array}$ & 15 \\
\hline Desde una asociación empresarial & 3 \\
\hline Desde la empresa matriz & 13 \\
\hline $\begin{array}{l}\text { Desde agencias de desarrollo regional o } \\
\text { centros tecnológicos }\end{array}$ & 8 \\
\hline
\end{tabular}

Tabla 8. Servicios de información sobre patentes utilizados por las empresas

Al realizar el análisis en función de los sectores económicos se pone de manifiesto que los sectores de ingeniería civil-bienes de consumo, electricidad-electrónica, ingeniería de proceso e ingeniería mecánica y maquinaria utilizan en mayor medida a los agentes de propiedad industrial y a la Oficina Española de Patentes y Marcas como servicios de información. Por su parte, el sector instrumentación, aunque también utiliza estos servicios, se apoya más en los recursos de acceso directo (cd-rom, internet), mientras que en química-farmacia se utilizan con elevada frecuencia las tres opciones de forma indistinta.

\subsection{Proveedores de bases de datos o servicios en in- ternet utilizados}

La principal fuente directa de acceso a los servicios de información sobre patentes es mediante internet a la 
base de datos Espacenet, según un 24\% de las respuestas; si bien un $17 \%$ de las empresas prefieren el proveedor comercial Derwent (World Patent Index) y un $13 \%$ utiliza los servicios de $I B M$ (que han experimentado un gran crecimiento en los últimos años) y la base de datos Oepmpat. Por el contrario, el otro gran proveedor comercial europeo de servicios de información sobre patentes Questel-Orbit apenas es utilizado por las empresas españolas (4\%), al igual que el sistema Dialog (7\%) (tabla 9).

\begin{tabular}{|l|c|}
\hline \multicolumn{1}{|c|}{ Proveedor/Servicio } & \% \\
\hline Espacenet & 24 \\
\hline Oepmpat & 13 \\
\hline Cibepat & 7 \\
\hline Derwent & 17 \\
\hline Questel-Orbit & 4 \\
\hline STN & 10 \\
\hline Micropatent & 3 \\
\hline IBM & 13 \\
\hline Dialog & 7 \\
\hline Reuter & 1 \\
\hline
\end{tabular}

Tabla 9. Bases de datos y servicios en internet utilizados por las empresas

El mismo esquema de utilización presenta el análisis de las empresas en función de su tamaño, si bien se puede resaltar que las grandes prefieren el proveedor Derwent (25\%) a la base de datos Espacenet (23\%).

Cuando el análisis se realiza en función de los sectores económicos se pone de relieve que el sector de química-farmacia es el que hace mayor uso del proveedor Derwent, mientras que la base de datos Oepmpat es requerida principalmente en ingeniería de proceso e ingeniería civil-bienes de consumo. Las compañías del resto de sectores (instrumentación, electricidad-electrónica e ingeniería mecánica y maquinaria) utilizan más la base de datos Espacenet.

\subsection{Incremento del uso de los documentos de paten- tes como fuente de información}

Una gran parte de las sociedades que tienen patentes $(62 \%)$ expresa su intención de empezar a utilizar o incrementar el uso de los documentos de patentes como fuente de información en un futuro próximo (vigilancia tecnológica), mientras que el 38\% responde negativamente a la misma cuestión, debido principalmente a que ya utilizan sistemas de búsqueda. Son las empresas que tienen más de 50 empleados las que expresan con mayor claridad su deseo de incrementar este uso, lo que pone de manifiesto el interés en apoyarse en la patente como estrategia específica para impulsar y consolidar el proceso de innovación. Desde la perspectiva de los sectores económicos es el de química-farmacia el que en mayor medida expresa ese interés.

\section{"La innovación tecnológica no resulta fácilmente medible"}

\subsection{Factores que pueden impulsar a hacer más efec- tiva la búsqueda de información en las patentes}

Esta pregunta es complementaria a la anterior y las respuestas señalan que sería posible fomentar un mayor uso de las patentes como fuente de información tecnológica si se consiguiera una mayor facilidad de acceso directo a las mismas desde internet (48\%) y llevaran asociado una mayor cantidad de información (42\%). También se reconoce, aunque en menor medida, que a ello contribuiría de forma positiva una mayor accesibilidad a los documentos de patentes $(37 \%)$. Se observa que hay coincidencia en las respuestas por parte de las empresas que tienen más de 50 empleados, mientras que aquellas de menor tamaño no valoran tanto esta facilidad de acceso, sino los precios.

El análisis por sectores económicos refleja un perfil semejante al global, a excepción de las compañías de ingeniería de proceso que, en lugar de mayor nivel de información, exigen descuentos por mayor uso, lo que es coherente con el nivel de información que se obtiene de las solicitudes de patentes, más enfocado al producto que al proceso (tabla 10).

\begin{tabular}{|l|c|}
\hline \multicolumn{1}{|c|}{ Factores } & \% \\
\hline Precios más flexibles & 26 \\
\hline Descuentos por mayor uso & 11 \\
\hline Mayor facilidad de acceso & 37 \\
\hline Mayor nivel de cualificación en el personal & 11 \\
\hline Acceso directo desde internet & 49 \\
\hline Mayor nivel de información & 42 \\
\hline
\end{tabular}

Tabla 10. Factores que pueden impulsar o hacer más efectiva la búsqueda de información en las patentes

\subsection{Importancia de otras formas de propiedad in- dustrial para el avance de la actividad empresarial}

Si bien el proyecto se basa en el análisis del papel que desempeñan las patentes como elemento de referencia para identificar patrones de innovación empresarial, resulta interesante conocer si las empresas españolas 
que tienen patentes utilizan además otros instrumentos de propiedad industrial.

En relación con este aspecto, las empresas consideran que también hay otras formas de propiedad industrial que son relevantes para incrementar la actividad innovadora y que complementan en buena medida a la patente como instrumento específico. Estas son las marcas $(76 \%)$ y los modelos de utilidad (56\%). Por el contrario, el copyright (11\%), los secretos industriales $(17 \%)$ y los dibujos industriales $(20 \%)$ son considerados importantes por un reducido número de empresas, en su mayor parte por aquellas que tienen menos de 250 empleados.

En función del tamaño, las grandes compañías utilizan principalmente las opciones de los dibujos industriales $(25 \%)$ y los secretos industriales $(30 \%)$ como consecuencia de su mayor capacidad innovadora y la fuerte competencia a la que están sometidas. También, resalta el hecho de que sean las más pequeñas (5-10 empleados), las que conceden mayor relevancia al copyright como forma eficaz de protección (33\%) (tabla 11).

El análisis por sectores económicos refleja la consolidación de la marca y los modelos de utilidad como formas de protección de la propiedad industrial más utilizadas y valoradas. Tan sólo destaca el sector ingeniería de proceso que concede igual importancia relativa al modelo de utilidad y al dibujo industrial. Por su parte, los secretos industriales son más valorados en química-farmacia.

\begin{tabular}{|l|c|c|c|c|c|}
\hline \multirow{2}{*}{ Opciones } & \multicolumn{5}{|c|}{ Tamaño de la empresa } \\
\cline { 2 - 6 } & $\mathbf{5 - 1 0}$ & $\mathbf{1 1 - 5 0}$ & $\begin{array}{c}\mathbf{5 1 -} \\
\mathbf{1 0 0}\end{array}$ & $\begin{array}{c}\mathbf{1 0 1 -} \\
\mathbf{2 5 0}\end{array}$ & $\mathbf{7 2 5 0}$ \\
\hline Marcas & 56 & 88 & 74 & 80 & 75 \\
\hline $\begin{array}{l}\text { Modelos de } \\
\text { utilidad }\end{array}$ & 33 & 25 & 52 & 60 & 68 \\
\hline $\begin{array}{l}\text { Dibujos } \\
\text { industriales }\end{array}$ & 11 & 0 & 22 & 20 & 25 \\
\hline $\begin{array}{l}\text { Secretos } \\
\text { industriales }\end{array}$ & 33 & 6 & 9 & 4 & 30 \\
\hline Copyright & 33 & 0 & 9 & 8 & 16 \\
\hline
\end{tabular}

Tabla 11. Importancia de otras formas de propiedad industrial para las empresas (\%)

\section{Conclusiones}

En las dos últimas décadas se ha puesto de manifiesto que la innovación tecnológica es un proceso complejo que influye de manera determinante en la capacidad de producir nuevos productos y, por tanto, constituye un elemento esencial para comprender el nivel de desarrollo tecnológico de un país. No obstante, la realidad es que no resulta fácilmente medible, aunque está contrastada su relación con indicadores asociados a los procesos de investigación y desarrollo (I+D) y con la existencia de capacidades tecnológicas.

\section{"Los servicios de información sobre patentes más utilizados son los agentes de la propiedad industrial y la Oficina Española de Patentes y Marcas"}

Del conjunto de indicadores disponibles, aquellos que más información aportan sobre los resultados de los procesos de innovación son las patentes, si bien su uso también se enfrenta a un conjunto de limitaciones que son superadas por las ventajas que conllevan. La principal novedad que incorpora este análisis se encuentra en la identificación de los elementos cualitativos que aportan las solicitudes de patentes y que no han sido utilizados en la mayor parte de los análisis realizados en España con base en estos indicadores.

El nivel de utilización de la información descrita por las patentes pone de relieve que se les concede una gran importancia fundamentalmente como instrumento de defensa de la propiedad industrial y, en menor medida, como instrumento que proporciona a la empresa mayor capacidad de negociación con otras compañías. Tan sólo el segmento de grandes empresas considera importantes las patentes como instrumento de ayuda a la exportación de tecnología y a la formalización del conocimiento.

Desde la perspectiva de la frecuencia en el uso de los servicios de información sobre patentes se obtiene que las sociedades de tamaño medio y grande los utilizan de forma mensual, mientras que las pequeñas y micro empresas tienen una frecuencia inferior. La principal finalidad en el uso de los servicios de información sobre patentes es la realización de búsquedas previas al comienzo de un proyecto de I+D en la organización, evitando invertir en algo ya existente, y también la obtención de información acerca de los avances de los competidores.

Entre los servicios de información sobre patentes más utilizados se encuentran los agentes de la propiedad industrial y la Oepm. Otras opciones como centros tecnológicos y asociaciones empresariales apenas tienen valoración.

La principal fuente directa de acceso a los servicios de información sobre patentes es la base de datos Espacenet accediendo directamente por internet, y también el proveedor comercial Derwent. Por último, se pone de manifiesto que sería posible fomentar en mayor me- 
dida el uso de las patentes como fuente de información tecnológica si se consiguiera un acceso directo más fácil desde internet.

\section{Nota}

Para mayor claridad los porcentajes que figuran en este artículo se han redondeado

\section{Bibliografía}

Archibugi, Daniele. "Patenting as an indicator of technological innovation: a review". Science and public policy. 1992, December, v. 19, n. 6, pp. $357-$ 368.

http://www.danielearchibugi.org/downloads/papers/Archibugi_Patenting _ S\&PP_92.pdf

Arundel, Anthony; Kabla, Isabelle. "What percentage of innovations are patented? Empirical estimates for European firms". Research policy, 1998, v. 27, n. 2 , pp. $127-141$

Basberg, Bjorn. "Patents and the measurement of the technological change: a survey of the literature". Research policy. 1987, v. 16, n. 2-4, p. 131141.

Díaz-Pérez, Maidelyn; De-Moya-Anegón, Félix. "El análisis de patentes como estrategia para la toma de decisiones innovadoras". El profesional de la información, 2008, mayo-junio, v. 17, n. 3, pp. 293-302.

Dosi, Giovanni. "Sources, procedures, and microeconomics effects of innovation". Journal of economic literature, 1988, September, v. 26, n. 3, pp. 1120-1171.

Escorsa, Pere; Maspons, Ramón. De la vigilancia tecnológica a la inteligencia competitiva. Madrid: Prentice Hall, 2001.

Freeman, Chris; Soete, Luc. The economics of industrial innovation. London, Frances Pinter, 1982

Glazier, Stephen. Patent strategies for business. USA, Law \& Business Institute, 2003.

Griliches, Zvi. "Patent statistics as economic indicators: a survey". Journal of economic literature, 1990, December, v. 17, pp. 1661-1707.

Hidalgo, Antonio; Molero, José. "Technology and growth in Spain (19501960): an evidence of Schumpeterian pattern of innovation based on patents". World patent information, in press, v. 31, n. 3, September 2009, pp. 199-206.

Kline, Stephen; Rosenberg, Nathan. "An overview of innovation". En: Landau, Ralph; Rosenberg, Nathan. The positive sum strategy. Washington DC: National Academy Press, 1986, pp. 273-305.
Muñoz-Durán, Javier; Marín-Martínez, María; Vallejo-Triano, José. "La vigilancia tecnológica en la gestión de proyectos de $\mathrm{I}+\mathrm{D}+\mathrm{i}$ : recursos y herramientas". El profesional de la información, 2006, noviembre-diciembre, v. 15 , n. 6 , pp. 411-419.

Narin, Francis; Norma, Elliot; Perry, Ross. "Patents as indicators of corporate technological strength". Research policy. 1987, v. 16, pp. n. 2-4, pp. 143-155.

Newton, David. How to find information: patents on the internet. Great Britain: The British Library, 2000.

OCDE. Oslo manual. Guidelines for collecting and interpreting innovation data. Paris: Oecd, Eurostat, European Commission, 2005.

Pavitt, Keith. "Do patents reflect the useful research output of universities?". Research evaluation, 1998, August, v. 7, n. 2. pp. 105-112.

Rojo, Raquel; Gómez, Isabel. "Análisis de la producción científica y tecnológica de la industria española en el sector de las tecnologías de la información y de las comunicaciones". El profesional de la información, 2006, mayo-junio, v. 15, n. 3, pp. 190-201.

Schmookler, Jacob. Invention and economic growth. Cambridge (MA): Harvard University Press, 1966.

\section{Agradecimientos}

Los autores agradecen su colaboración en la realización de este análisis a la Oficina Española de Patentes y Marcas mediante el proyecto Análisis estadístico de datos básicos de patentes como indicadores de innovación.

Antonio Hidalgo-Nuchera, Depto. de Ingeniería de Organización, Administración de Empresas y Estadística, Escuela Técnica Superior de Ingenieros Industriales, Univ. Politécnica de Madrid.

ahidalgo@etsii.upm.es

Santiago Iglesias-Pradas y Ángel Hernández-García, Depto. de Ingeniería de Organización, Administración de Empresas y Estadística, Escuela Técnica Superior de Ingenieros de Telecomunicación, Univ. Politécnica de Madrid.

s.iglesias@upm.es

angel.hernandez@upm.es

\section{Spanish Meeting Point}

La revista El profesional de la información organiza una nueva edición del Spanish Meeting Point, dentro de la Online Information Conference, en Londres - la más importante del mundo en materia de contenidos y bases de datos científicas y profesionales, así como en software de gestión de contenidos.

Online Information Conference, Londres 1-3 diciembre 2009 http://www.online-information.co.uk

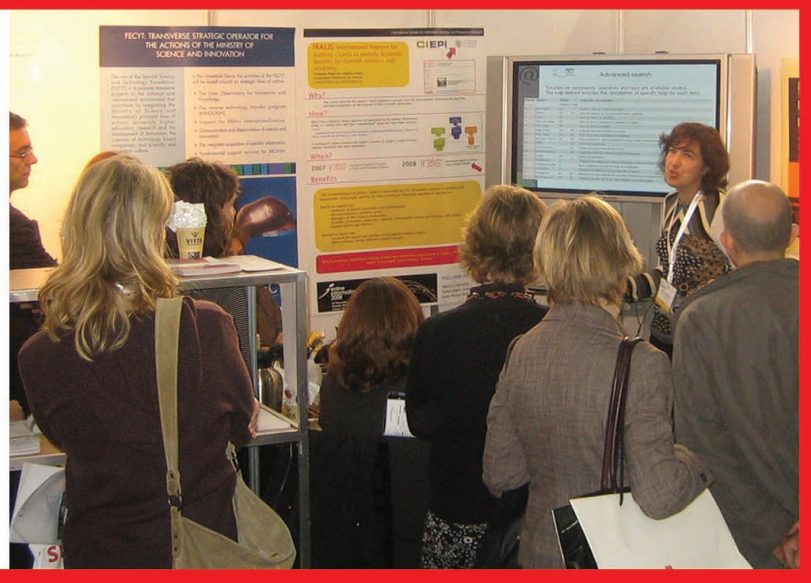


